\title{
Dependence of Neighbor Data Sequences Based on Voltage Mode Controlled Boost Converter
}

\author{
Xitong Zhu ${ }^{\mathrm{a}}$, Yan Luo ${ }^{\mathrm{b}}$, Nuo Zhou ${ }^{\mathrm{c}}$ and Hongmei $\mathrm{Xu}^{\mathrm{d}^{*}}$ \\ Institute of Engineering Yanbian University, Yanji, China \\ a1518788773@qq.com,,luoyan015@163.com, '821272123@qq.com, dhmxu@ybu.edu.cn \\ * The corresponding author
}

Keywords: Boost converter; Neighbor data; Dependence; Chaos

\begin{abstract}
The original complicated array is converted into a simple distance of neighbor sequences based on the distance variation of boost converter, the chaotic state of boost converter can be detected obviously throughout computing Euclidean distance, the application potential of this method is worthy of great attention.
\end{abstract}

\section{Introduction}

Voltage mode controlled Boost converter has been widely used in communication, military, computer, instrumentation and other fields[1-2]. According to the working mode of inductor current in Boost converter, it can be divided into continuous inductance current mode $(\mathrm{CCM})$ and discontinuous inductor current mode(DCM). Electronic engineers have been exploring effective method of the topology transformation and improving the working performance. Zhanybai studied the tours birth bifurcation in a DC/DC converter[3]. Porporato studied irreversibility and fluctuation theorem in stationary time series[4]. Xie researched bifurcation and border collision analysis of voltage-mode-controlled flyback converter based on total ampere-turns[5]. Literature[6] researched on parameter estimating of chaotic systems via symbolic time series analysis.

Recent years always use the qualitative analysis of switching converter chaos characteristics, at the same time quantitative measurement methods have been proposed, such as lyapunov, fractal dimension and entropy theory [7-9]. In the practical application, phase space reconstruction can not fully reproduce the real phase space features, the scale free zone identification is also needed to be further improved due to many influencing factors. This article putting forward a kind of chaotic analysis method based on adjacent data dependence under complex data sequence. This method convert complicated data column to simpler distance sequence by measuring the change of one dimensional data subsequence distance values of switching converter, avoids phase space non scale region select procession. It can organic combine qualitative and quantitative analysis methods, distinctly separate different chaotic states of switching converters, and this could provide an effective analysis method for switching converter analysis and design.

\section{The Analysis Method of Data Relevance}

Analysis the characteristics by calculating the numerical variation of euclidean distance of adjacent equal length subsequences in one-dimensional data sequence. For data sequence $\left\{x_{n}\right\}$, using Eq.1 to calculate distance:

$$
d_{i}=\left|X_{i+1}-X_{i}\right|=\left(\sum_{j=0}^{m-1}\left(x_{i+1+m}-x_{i+m}\right)\right)^{1 / 2}
$$

In the equation, $i \leq n-m, d_{i}$ is the distance of adjacent subsequence. According to the change of value $i$, calculate the distance of adjacent subsequence in turn, and compose a new sequence $\left\{x_{d}\right\}$. Calculating the mean and standard deviation of new sequence to analyze characteristic.

In order to analyze the data dependence, we can use the change rate and change range of adjacent 
points for further analysis. As Eq.2 and Eq.3:

$$
\begin{aligned}
& R_{j}=\left|d_{j+1}-d_{j}\right| / d_{j} \\
& F_{j}=\left|d_{j+1}-d_{j}\right|
\end{aligned}
$$

In the equations, $j \in[1, L], L$ is the length of adjacent subsequence, the change rate and change range form new sequence. The trend of the two changes can further analyze the data dynamic characteristics.

\section{Voltage Controlled Boost Converter Discrete Modeling and Relevance Analysis}

This article mainly analysis data relevance base on the switching converter modeling that proposed by TSE [10]. According to the Boost converter schematic diagram shown in Fig. 1. Let $E$ as input voltage, let $X$ as desired steady-state output voltage, let $D$ as steady-state duty cycle, let $k$ as feedback proportional gain. The working state of the converter can be described by the following iterative mapping equation.

$$
x_{n+1}=f\left(k, x_{n}\right)=\alpha x_{n}+\frac{\beta h\left(d_{n}\right)^{2} E^{2}}{x_{n}-E}
$$

In the equation, $x_{n}$ represents the $\mathrm{n}$ th times capacitor voltage when the inductor current is 0 , other parameters are as follows:

$$
\begin{aligned}
& \alpha=1-\frac{T}{R C}+\frac{T^{2}}{2 R^{2} C^{2}} \\
& \beta=\frac{T^{2}}{2 L C} \\
& h\left(d_{n}\right)= \begin{cases}0, & d\left(x_{n}\right)<0 \\
1, & d\left(x_{n}\right)>1 \\
d\left(x_{n}\right), & \text { others }\end{cases} \\
& d\left(x_{n}\right)=D-k\left(x_{n}-X\right)
\end{aligned}
$$

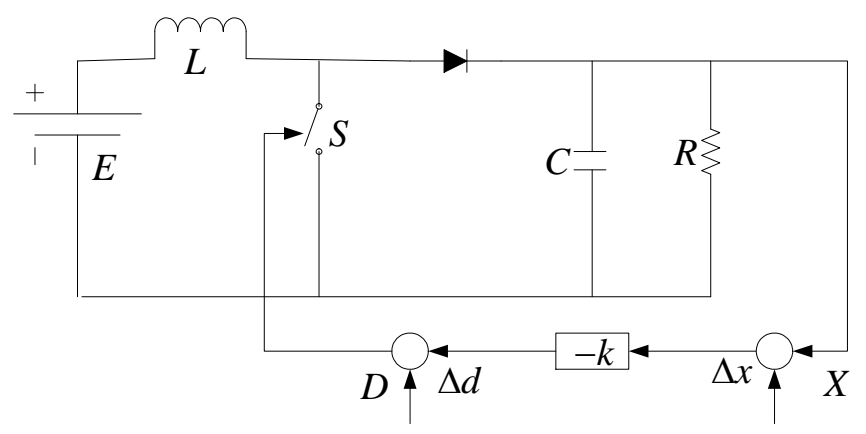

Figure 1. Voltage feedback boost converter schematic diagram

The parameters of the converter are shown in Table 1. Set feedback gain $k$ as the variable, while $k \in[0.01,0.14]$, taking 100 intervals, select initial value 99 times from 23.7 to 29.8, Boost converter bifurcation diagram is obtained in Fig. 2.

Set feedback gain $k$ as variable and take a hundred values of $\mathrm{k}$ from 0.0707 to 0.1400 , according 
to Eq.4 obtained a iteration sequence that data length is 5000. Remove the first 1000 transient data of the original sequence, then according to the Eq.1 calculating the adjacent subsequence distance (the length of subsequence is 2000 ) composed adjacent subsequence distance sequence. Calculating the mean value and standard deviation of the adjacent distance sequence respectively, as shown in Fig. 3 and Fig. 4.

Table 1 Boost converter parameter

\begin{tabular}{cc||cc}
\hline Parameter & Values & Parameter & Values \\
\hline $\operatorname{period} T[\mu s]$ & 333.33 & Duty cycle $D$ & 0.2874 \\
\hline Capacitance $C[\mu F]$ & 222 & Inductance & 208 \\
\hline Load $R[\Omega]$ & 12.5 & $L[\mu H]$ & 0.8872 \\
\hline$\beta$ & 1.2 & $\alpha$ & \\
\hline
\end{tabular}

It can be concluded from the change of adjacent distance mean values: the adjacent distance value increases with the smooth curve when the switching converter appears bifurcation phenomenon. When the switch come into chaotic state, the mean value oscillation increases with $\mathrm{k}$ increased. As show in the standard deviation value change of adjacent distance, the standard deviation value obviously greater than 0 when switch converter come into chaotic state, and shown growth trend in the significant fluctuation. Compared with the bifurcation diagram, standard deviation value can quantify the change of chaotic characteristic clearly.

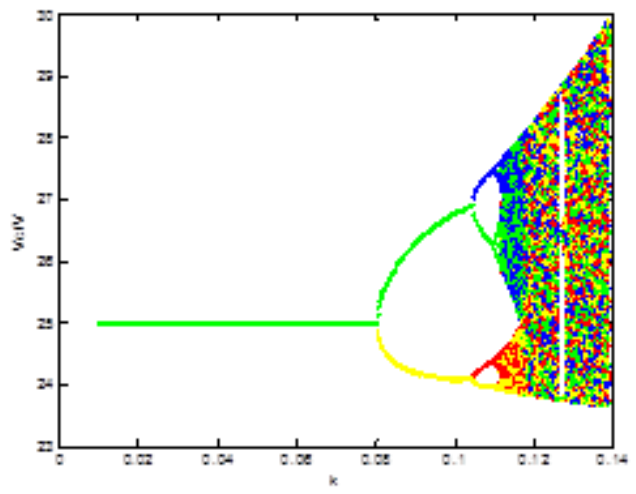

Figure 2. Voltage feedback boost converter bifurcation diagram

For the adjacent distance mean sequence, the change rate and the change range of the adjacent points could be calculated by the Eq.2 and the Eq.3, as shown in Fig .5 and Fig .7. Because the adjacent point change rate of the adjacent distance mean sequence is very big at Boost converter bifurcation point, and make the behind change rate close to 0 , so intercept 58 values of $\mathrm{k} \in[0.1001,0.1400]$ and local enlarge Fig .5 ,are shown as Fig. 6. It can be concluded from the two figures that when the switching converter come into chaos state, the change rate and the change range will appear obvious fluctuation. So the analysis method based on adjacent data relevance has the quantify ability, and more sensitive to the change of chaotic characteristics, and more clear to the identification of chaotic state self-similar structure in the switching converter. 


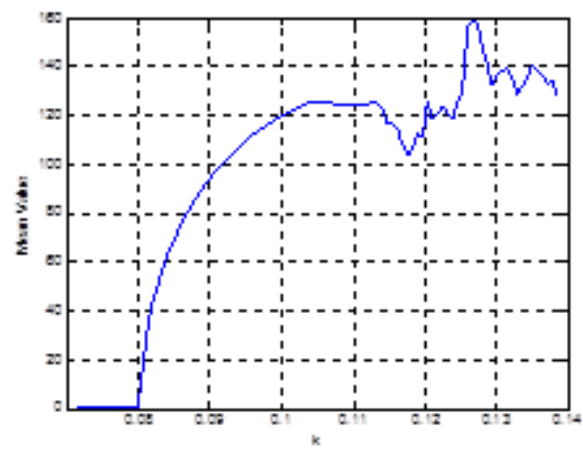

Figure 3. Adjacent distance sequence mean value change curve of voltage feedback Boost converter $(k \in[0.0707,0.1400])$

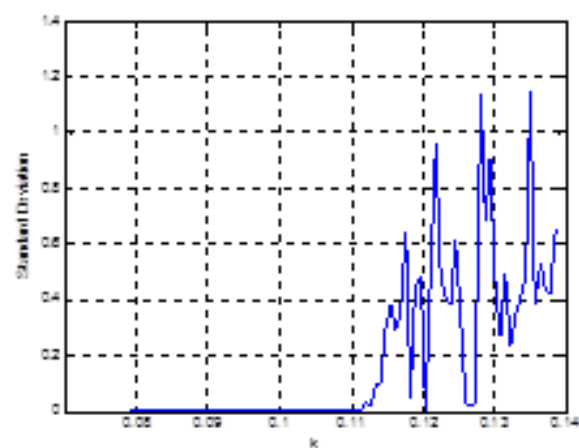

Figure 4. Adjacent distance sequence standard deviation value change curve of voltage feedback Boost converter $(k \in[0.0707,0.1400])$

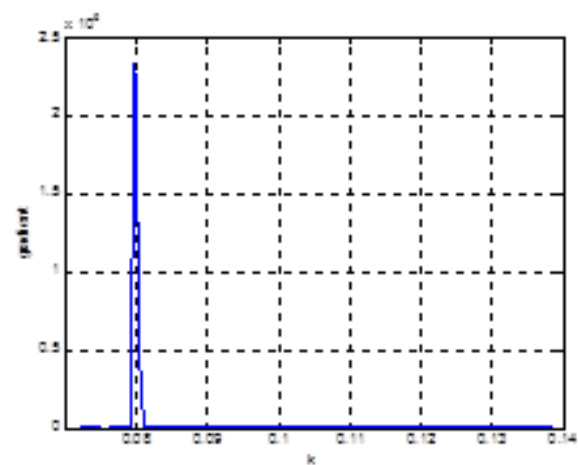

Figure 5. Adjacent point change rate of adjacent distance mean sequence of voltage feedback Boost converter $(k \in[0.0707,0.1400])$

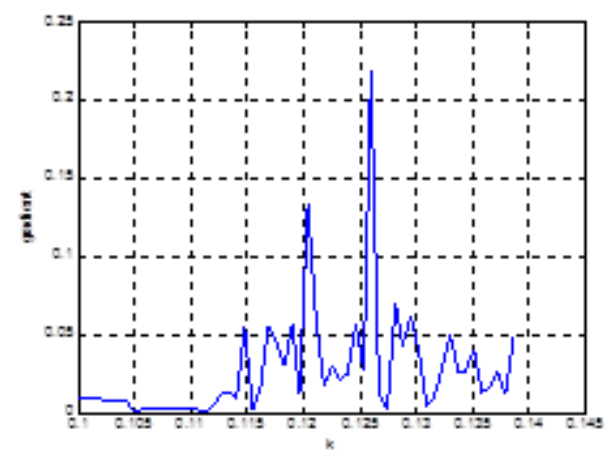

Figure 6. Adjacent point change rate local enlarged image $(k \in[0.1001,0.1400])$ 


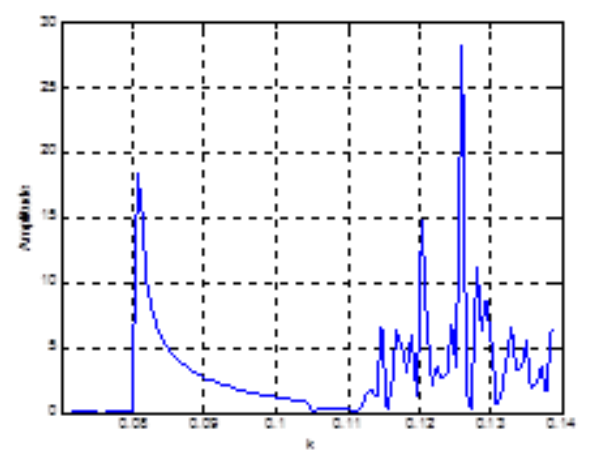

Figure 7. Adjacent point change range of adjacent distance mean sequence of voltage feedback Boost converter $(k \in[0.0707,0.1400])$

\section{Conclusions}

In this paper, the voltage controlled Boost converter is taken as an example, converting one-dimensional time series to distance value of an adjacent data sequence, and obtain follow conclusions by analysis data sequence relevance: The mean of the adjacent distance can clearly describe the process form doubling bifurcation period to the chaotic state, the adjacent distance standard deviation could identify and quantify the chaotic state, and multi period structure can be clearly distinguished from the chaotic state. The change rate and change range of data point in the adjacent distances mean sequence could further perfect the qualitative and quantitative analysis of switch converter. Compared to the traditional analysis method, this method have certain anti noise ability and avoid the problem of phase space reconstruction. At the same time, it can both consider the qualitative and quantitative analysis methods, identify the chaotic state more convenient.

\section{References}

[1] Hao Zhang, Xiaoping, Yang, Xikui Ma, and Bo He, Nonlinear Analysis: Real World Applications, 2012,01,002.

[2] Xuemei Wang, Bo Zhang, and Dongyuan Qiu, IEEE Trans. Power Electron, 2011, 26, 2101.

[3] Zhanybai T. Zhusubaliyev and Erik Mosekilde, IEEE Trans. Circuit and Syst. I, 2006,53,1839.

[4] A,Porporato, J.R. Rigby, and E, Daly, Phys. Rev. Lett,2007,98,094101.

[5] Fan Xie, Ru Yang, and Bo Zhang, IEEE Trans. Circuit and Syst. I, 2011,58,2269.

[6] Carlo Piccardi, CHAOS, 2006,16, 043115.

[7] Madalena Costa, Ary L. Goldberger, and C.K.Peng, Phys. Rev. Lett, 2002, 89,068102.

[8] D. Andrieux, and P. Gaspard, Phys. Rev. Lett, 2007, 98,150601.

[9] L.F. Santos, F.Borgonovi, and E.M.Izrailev, Phys. Rev. Lett, 2012,108,094102.

[10]C K Tse, and K.M.Adams, IEEE Trans. Power Electron, 1990, 5,323. 